

\title{
Distribution of conformational states as common source of g-and A-strain in the ESR spectra of proteins and glasses
}

Salvatore Cannistraro

\section{- To cite this version:}

Salvatore Cannistraro. Distribution of conformational states as common source of g-and A-strain in the ESR spectra of proteins and glasses. Journal de Physique, 1990, 51 (1), pp.131-139. 10.1051/jphys:01990005101013100 . jpa-00212348

HAL Id: jpa-00212348

https://hal.science/jpa-00212348

Submitted on 1 Jan 1990

HAL is a multi-disciplinary open access archive for the deposit and dissemination of scientific research documents, whether they are published or not. The documents may come from teaching and research institutions in France or abroad, or from public or private research centers.
L'archive ouverte pluridisciplinaire $\mathbf{H A L}$, est destinée au dépôt et à la diffusion de documents scientifiques de niveau recherche, publiés ou non, émanant des établissements d'enseignement et de recherche français ou étrangers, des laboratoires publics ou privés. 
Classification

Physics Abstracts

$76.30-61.40-87.15$

\title{
Distribution of conformational states as common source of $g$ - and $A$-strain in the ESR spectra of proteins and glasses
}

\author{
Salvatore Cannistraro \\ Gruppo di Biofisica Molecolare, Dipartimento di Fisica dell'Università, I-06100 Perugia, Italy
}

(Reçu le 9 mars 1989, révisé le 9 août 1989, accepté le 15 septembre 1989)

\begin{abstract}
Résumé. - On décrit quelques faits expérimentaux supplémentaires concernant la similitude structurale et dynamique entre protéines et verres, à partir de l'analyse de spectres ESR de quelques protéines contenant du cuivre et de verres dopés au $\mathrm{Cu}^{++}$formé par de l'eau et un deuxième composant ( $\mathrm{NaOH}, \mathrm{DMSO}$, alcools), obtenus à différentes températures. Tous les spectres obtenus à des températures inférieures à environ $210 \mathrm{~K}$ montrent des déformations $g$ et $A$ significatives qui consistent en un élargissement progressif et une réduction d'intensité des lignes hyperfines du cuivre, quand l'ordre de $m_{I}$ augmente (le spin nucléaire du cuivre est $I=3 / 2$ ). Ces faits sont pris en compte dans un modèle théorique qui attribue la distribution aléatoire des champs électriques autour des ions de cuivre à une distribution des énergies de conformation aussi bien pour les protéines que pour les matériaux amorphes.
\end{abstract}

\begin{abstract}
Additional experimental evidence on the structural and dynamical similarity between proteins and glasses is provided by the analysis conducted on the ESR spectra, at different temperatures, of some copper proteins and of some $\mathrm{Cu}^{++}$-doped glasses formed by water and a second component (NaOH, DMSO, alcohols). All the spectra taken at temperatures below $\sim 210 \mathrm{~K}$ display a significant $g$ - and $A$-strain that consists in a progressive broadening and decrese in the intensity of the copper hyperfine lines as the order of $m_{I}$ increases $(I=3 / 2$ is the $\mathrm{Cu}$ nuclear spin). These spectral features are taken into account by using a theoretical model which attributes the random distribution of the electric ligand fields around the copper ions to a distribution of the conformational substate energies both in the proteins and in the amorphous materials.
\end{abstract}

\section{Introduction.}

There is mounting evidence that biomolecules and glasses display profound analogies in their structure and dynamics [1-9]. At low temperatures amorphous solids exhibit characteristic anomalies in their thermal, acoustic and dielectric properties. These anomalies are attributed to low-energy excitations which are generally described as two level tunnelling systems (TLS) [10]. In a simple microscopic model, these excitations are thought to arise from the possibility that in an amorphous network small groups of atoms can undergo local rearrangements visualized by a transition between two wells of a double well potential.

On the other hand, biomolecules have been shown to exist in very many different, thermally accessible, configurational states (substates) that are believed to play an important dynamical role connected with their functionality. On cooling below $\sim 200 \mathrm{~K}$ the various 
molecules freeze into different substates, each characterized by a slightly different energy, transitions among these substates being absent. In that context, the characteristics of glasses and spin glasses find a correspondence in biomolecules : below a critical temperature there exist a large number of energy valleys separated by effectively infinitely high barriers, the system is non-ergodic and properties vary from valley to valley (substate to substate). The substates possess a hierarchical structure and relaxation is sequential and non-exponential in time as suggested for glasses (see Refs. [2, 8] and Refs. therein). In particular, it has been suggested that the glass-like states in proteins could be due to local arrangements of certain atoms or groups of atoms (for example, rearrangements of hydrogen bonds and rotations of methyl or similar groups) $[3,4,9]$ and that hydration water could play a crucial role in the dynamics of conformational transitions $[1,4,7]$.

More experimental evidence on the similarity between proteins and glasses is provided, in the present paper, by the analysis conducted on the Electron Spin Resonance (ESR) spectra, at different temperature, of some copper proteins and of some ${ }^{65} \mathrm{Cu}^{2+}$-doped glasses formed by water and a second component ( $\mathrm{NaOH}, \mathrm{DMSO}$, alcohols) [11].

All the spectra taken at temperatures below $210 \mathrm{~K}$ display a significiant $g$ - and $A$-strain that consists in a progressive broadening and decrease in the intensity of the copper hyperfine lines as the order of $m_{I}$ increases ( $I=3 / 2$ is the $\mathrm{Cu}$ nuclear spin) $[12,13]$. These spectral features have been almost perfectly reproduced by using a theoretical model that attributes the random distribution of the electric ligand fields around the copper ions, which is responsible for the statistical modulation of the $g$ and $A$ values, to a distribution of the conformational substate energies both in the proteins and in the amorphous materials.

\section{Materials and methods.}

Plastocyanin (PC) was prepared from spinach leaves as described in reference [14] Azurin (Az), from Pseudomonas Aeruginosa, was purchased from Sigma Chem. Co. and used without further purification. ${ }^{65} \mathrm{Cu}^{++}$was purchased from Oak Ridge Labs, digested in $\mathrm{HCl}$ and diluted in phosphate buffer. All the other chemical used were of analytical reagent grade.

${ }^{65} \mathrm{Cu}^{++}$-doped amorphous systems and related ESR samples were prepared with procedures similar to those reported in reference [15]. ESR protein samples were prepared according to procedures reported in reference [16].

ESR spectra were recorded by an X-band Varian E109 spectrometer, equipped with a variable temperature control. To calculate the experimental $g$-values, a magnetic field calibration was performed with a Magnion Precision NMR Gaussmeter mod. G-502, the microwave frequency being measured with a Marconi 2440 counter. ESR data acquisition was carried out on an HP 86A personal computer through a home made interface connected to an IEEE 488 bus [17]. To run simulation and best-fit programs, the same microcomputer was switched to an intelligent terminal of the main frame computer (a Prime 550-1), through an RS-232C serial interface and an HP terminal emulator.

The chi-square test was used to evaluate the goodness of fits utilizing the expression :

$$
\chi^{2}=\sum_{i=1}^{N}\left[\frac{I^{\exp }\left(H_{i}\right)-I^{\text {th }}\left(H_{i}, p\right)}{\sigma_{i}}\right]^{2}
$$

where $I^{\exp }\left(H_{i}\right)$ is the derivative of the experimental ESR absorption spectrum sampled at 200 discrete points of the field, $I^{\text {th }}\left(H_{i}, p\right)$ is the model function of equation (17) (see below) and $\sigma_{i}$ is the standard deviation calculated for the $\mathrm{i}$-th experimental point of the ESR spectrum by repeated runs. 


\section{Results and discussion.}

Figures 1a and 2a show the ESR spectra arising from frozen aqueous solutions of two wellknown metallo-proteins, $\mathrm{PC}$ and $\mathrm{AZ}$ respectively. Each of these two proteins contains one single atom of copper, which, under its oxidized form $\mathrm{CU}^{++}$, endows the protein with paramagnetic properties [18]. Actually, their spectrum, taken at $77 \mathrm{~K}$, displays the characteristic features of a powder-like ESR spectrum of $\mathrm{Cu}^{++}$ions with low $g_{\boldsymbol{\|}}$ and $A_{\boldsymbol{l}}$ values interpretable in terms of the following spin Hamiltonian :

$$
\mathcal{H}=\beta\left[g_{\|} H_{z} S_{z}+g_{\perp}\left(H_{x} S_{x}+H_{y} S_{y}\right)\right]+A_{l} I_{z} S_{z}+A_{\perp}\left(I_{x} S_{x}+I_{y} S_{y}\right)
$$

where $\beta$ is the Bohr magneton, $g_{\|}$and $A_{\|}$are, respectively, the $g$ value and the hyperfine component parallel to the molecular symmetry axis, $g_{\perp}$ and $A_{\perp}$ are, respectively, the $g$ value and hyperfine component perpendicular to the molecular symmetry axis, the $S$ 's $(S=1 / 2)$ are the electron spin components and $I$ 's $(I=3 / 2)$ are the nuclear spin components of the ion.

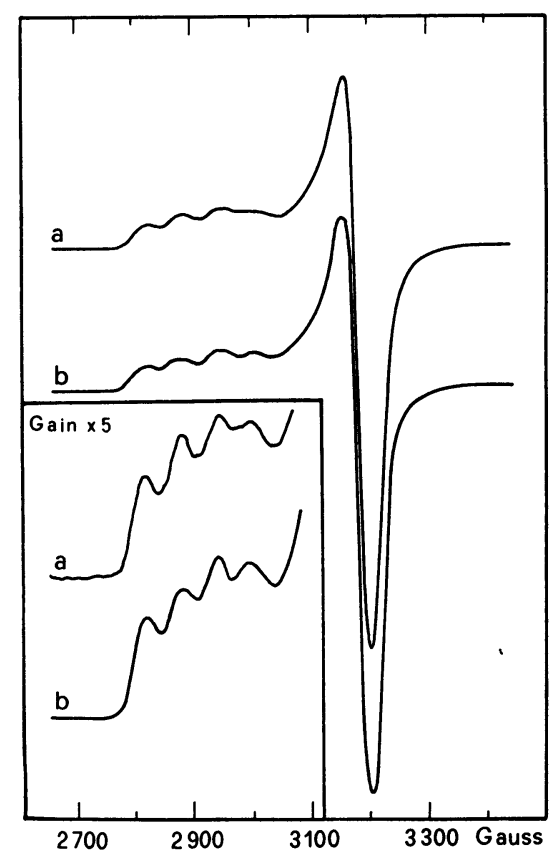

Fig. 1.

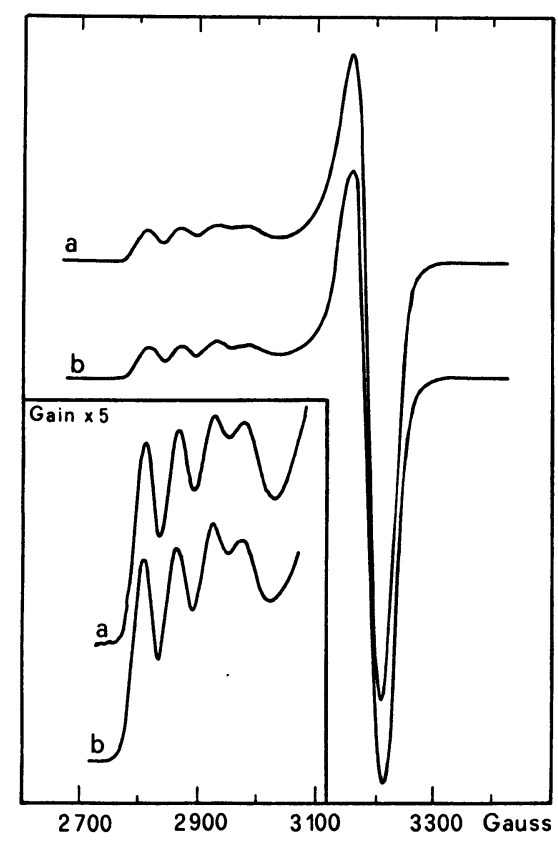

Fig. 2.

Fig. 1. - Experimental (a) and computer-simulated (b) ESR spectrum of PC (0.3 mM in water, $\mathrm{pH} 7)$. The low-field part $\left(g_{1}\right.$ region) of the spectra is shown at fivefold gain. The experimental spectrum was recorded at $77 \mathrm{~K}$ with the following settings : microwave power level : $20 \mathrm{~mW}$; magnetic field sweep rate $: 2000 \mathrm{G}$ in $8 \mathrm{~min}$; time constant $: 0.5 \mathrm{~s}$; modulation amplitude : $5 \mathrm{G}$. The computed spectrum was generated through equation(17) with the following parameters: $g_{1}=2.251 ; g_{\perp}=2.047$; $A_{\mid}=53.1 \mathrm{G} ; A_{\perp}=3.7 \mathrm{G} ; \sigma_{\nu}^{\mathrm{R}}=20.1 \mathrm{G} ; \sigma_{g \mid}=0.0074 ; \sigma_{A_{\mid}}=8.85 \mathrm{G} ; \rho=1$.

Fig. 2. - Experimental (a) and computer-simulated (b) ESR spectrum of Az (0.2 mM in water, $\mathrm{pH} 7)$. The low-field part ( $g_{1}$ region) of the spectra is shown at fivefold gain. Experimental temperature and settings are as in Fig. 1 . Simulated best fitting spectrum was obtained with : $g_{1}=2.275 ; g_{\perp}=2.056$; $A_{\mathrm{I}}=55.0 \mathrm{G} ; A_{\perp}=3.8 \mathrm{G} ; \sigma_{\nu}^{\mathrm{R}}=18.9 ; \sigma_{g \mid}=0.0069 ; \sigma_{\mathrm{Al}_{\mathrm{I}}}=7.88 \mathrm{G} ; \rho=1$. 
From the hyperfine coupling between $S$ and $I$, four lines centered at $g_{\|}$and separated by $A_{\|}$(related to $\left.m_{I}=-3 / 2,-1 / 2,1 / 2,3 / 2\right)$ and four lines centered at $g_{\perp}$ and separated by $A_{\perp}$ could be expected.

Qualitatively, a similar spectrum is also to be expected for $\mathrm{Cu}^{++}$ions dissolved in amorphous matrices as obtained by adding suitable amounts of $\mathrm{NaOH}$, DMSO and alcohols to $\mathrm{H}_{2} \mathrm{O}$ and by rapidly freezing the mixture below the glass transition [15]. Actually, this is the case and, as examples, in figures $3 \mathrm{a}$ and $4 \mathrm{a}$, we show the ESR spectra of ${ }^{65} \mathrm{Cu}^{++}$-doped solutions of $\mathrm{H}_{2} \mathrm{O}-\mathrm{DMSO}$ and $\mathrm{D}_{2} \mathrm{O}-\mathrm{NaOD}$, respectively, recorded at $77 \mathrm{~K}$ after the glass matrices were formed (isotopically enriched copper ions and deuterated chemical compounds were used to improve spectral resolution).

We would like to draw attention to a common feature shared by the spectra shown in the four figures. As already observed for some particular cases $[14,15]$, all the spectra of figures 1-4 show, in the $g_{\|}$region of the spectrum (this part is reproduced in the insets of the

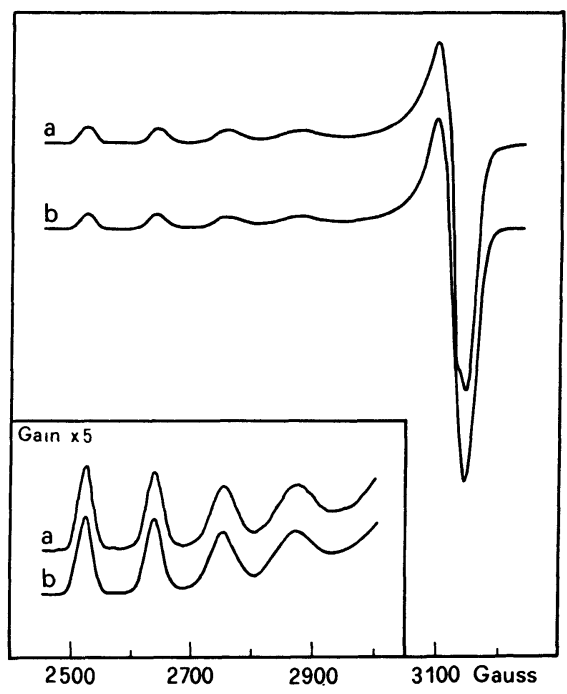

Fig. 3.

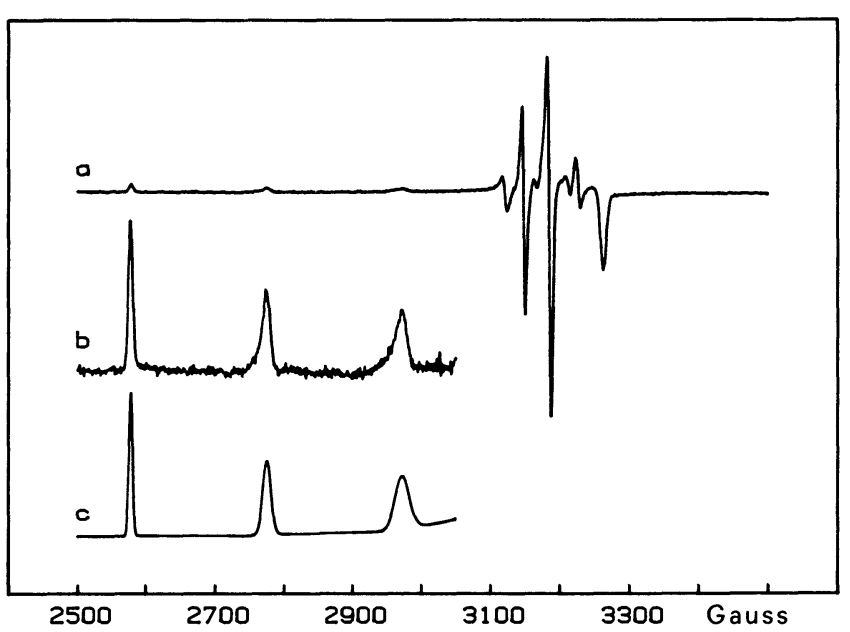

Fig. 4.

Fig. 3. - Experimental (a) and computer-simulated (b) ESR spectrum ${ }^{65} \mathrm{Cu}^{++}(0.1 \mathrm{mM})$ in waterDMSO (1:1, in vol). The low-field part ( $g_{1}$ region) of the spectra is shown at fivefold gain. The experimental spectrum was recorded at $77 \mathrm{~K}$ with the following settings : microwave power level : $12 \mathrm{~mW}$; magnetic field sweep rate : $2000 \mathrm{G}$ in $8 \mathrm{~min}$; time constant : $0.5 \mathrm{~s}$; modulation amplitude : $5 \mathrm{G}$. The computed spectrum was generated through equation (17) with the following parameters : $g_{\|}=2.2394 ; g_{\perp}=2.073 ; A_{\mid}=127.2 \mathrm{G} ; A_{\perp}=9.2 \mathrm{G} ; \sigma_{\nu}^{\mathrm{R}}=12.5 \mathrm{G} ; \sigma_{g_{\mathbf{l}}}=0.0180 ; \sigma_{A_{\mathbf{I}}}=8.5 ;$ $\rho=1$.

Fig. 4. - ESR spectrum from ${ }^{65} \mathrm{Cu}^{2+}$ doped $(0.01 \mathrm{mM}) \mathrm{NAOD}-\mathrm{D}_{2} \mathrm{O}$ in the amorphous phase (a) and its parallel part at a tenfold gain (b). Spectrum (c) is the best fit of all the experimental spectrum (fitting parameters : $g_{\mid}=2.271 ; g_{\perp}=2.031 ; A_{\mid}=197.6 \mathrm{G} ; A_{\perp}=40.0 \mathrm{G} ; \sigma_{g_{\mid}}=0.0062 \mathrm{G} ; \sigma_{A_{\mid}}=4.1 \mathrm{G}$; $\sigma_{\nu}^{\mathrm{R}}=2.5 \mathrm{G} ; \rho=1$ ). Only the $g$-parallel part (where the strain effects are mostly seen) of the simulated spectrum is shown to compare it to the related part od the experimental spectrum at high gain. The experimental spectrum was recorded at $77 \mathrm{~K}$, the other spectrometer settings being : microwave frequency : $9.133 \mathrm{GHz}$; microwave power ; $10 \mathrm{~mW}$; magnetic field sweep rate : $2000 \mathrm{G}$ in 8 min ; time constant : $0.5 \mathrm{~s}$; modulation amplitude $2 \mathrm{G}$. 
Fig. at a higher gain), a progressive braodening and decreasing in intensity of the hyperfine lines as $m_{I}$ increases. Such an effect can be immediately observed in the case of amorphous systems (Figs. 3,4) since the hyperfine lines are spaced to a higher extent than those arising from the metallo-proteins. In the ESR pattern of the latter systems, however, a closer inspection reveals a similar behaviour $[14,19]$. Moreover, an irregular spacing between pairs of adjacent hyperfine lines in the $g_{\|}$region is found $[12,13]$. Spectral anomalies of this kind have been named, in a general way, $g$ - and $A$-strain $[12,13,20,21]$. In the course of previous works $[12,13]$, we have shown that, under these conditions, a computer simulation of the experimental ESR spectra is indispensable if a reliable extraction of the spin hamiltonian parameters is to be achieved. For sake of completeness, it should be recalled that the $g$ strain effect in some classes of iron metalloproteins has been described in the literature with the aid of two different approaches : one taking into account a statistical fluctuation of some spin hamiltonian parameters (statistical model) [21-24] and the other expressing the $g$ values in terms of the related physical parameters (physical model) $[25,26]$.

In the present paper we would mainly like to point out that these features are present, with striking similarity, in the ESR spectra of both copper-proteins and of $\mathrm{Cu}^{++}$-doped glassy systems. In this connection, we have moreover attempted to relate these common anomalous spectral features to the microscopic, structural and dynamical, properties of both glassy and frozen protein systems.

Both proteins and glassy systems can be considered as microscopically disordered systems. This fact originates from fluctuations of local structure in glasses [10] and from the presence of a static ensemble of frozen conformational states, to which correspond randomly oriented or positioned side groups (see Introduction section), in the frozen protein solutions. As a consequence, we can imagine that the $\mathrm{Cu}^{++}$complexes are deformed (as regarding their bond length and angles) in a slightly different way according to the microenvironmental fluctuations that, very probably, follow a random distribution. The statistical distribution of the atomic coordinates that can be, then, envisaged for the copper ion ligands will result in a distribution of strains at the metal sites [21]. In the framework of the crystal field theory for the $3 \mathrm{~d}^{9} \mathrm{Cu}^{++}$ions [27], such a physical situation could be described by a distribution of the crystal field splittings $(\Delta)$ of the $\mathrm{d}^{9}$ levels of the $\mathrm{Cu}^{++}$ions both in the protein and in the glassy systems. In other words, it seems reasonable to imagine that the copper ions, present either as dopant in the amorphous matrices or as intrinsic constituents in the proteins, could sense the energy distribution corresponding to the existence of a multiplicity of conformational states as a statistical modulation of their crystal field strengths (in this respect, an Angular Overlap Model calculation has provided a quantitative relationship between the $\eta$ bond angle distribution [28] and the corresponding crystal field energy distribution for Az [29]).

The quite realistic assumption of a distribution of the conformational energies for the glasses and for the frozen solutions of proteins of the type [2,3]:

$$
P(E)=\frac{1}{\sqrt{2 \pi} \sigma_{E}} \mathrm{e}^{-\frac{\left(E-E_{0}\right)^{2}}{2 \sigma_{E}^{2}}}
$$

leads then to a Gaussian distribution of the crystal field splittings $\Delta$ (viewed, for example, as the energy difference between the $e_{g}$ and the $t_{2 g}$ orbitals) :

$$
P(\Delta)=\frac{1}{\sqrt{2 \pi} \sigma_{\Delta}} \mathrm{e}^{-\frac{\left(\Delta-\Delta_{0}\right)^{2}}{2 \sigma_{\Delta}^{2}}}
$$


If one now takes into account the Kivelson and Neiman formulas [30], written in a more compact way :

$$
g_{\|}=2.0023+a / \Delta ; \quad A_{\|}=b+c / \Delta
$$

where :

$$
a=-8 \lambda ; \quad b=P\left[-k-4 / 7+3 / 7\left(g_{\perp}-2.0023\right)\right] ; \quad c=P a=-8 P \lambda
$$

and $P, k$ and $\lambda$ have their usual meaning [30], it can be easily shown that, for small fluctuations in $\Delta$ (i.e. for $\left.\left|\left(\Delta-\Delta_{0}\right) / \Delta_{0}\right| \ll 1\right)$ the following expressions hold :

$$
g_{\|}=g_{\|}^{0}-a\left(\Delta-\Delta_{0}\right) / \Delta_{0}^{2} ; \quad A_{\|}=A_{\|}^{0}-c\left(\Delta-\Delta_{0}\right) / \Delta_{0}^{2}
$$

where :

$$
g_{\|}^{0}=2.0023+a / \Delta ; \quad A_{\|}^{0}=b+c / \Delta .
$$

In the derivation of equation (6), the fluctuation in the parameters $g_{\perp}$ and $A_{\perp}$, as resulting from the fluctuation in $\Delta$, has been neglected. Such an approximation can be made for two reasons : the first is related to the fact that, for these complexes, the strain effects are mostly seen in the $g$-parallel region (a similar approximation is made by other authors [20]), the second is based on the check that the inclusion in the model of the fluctuations in $g_{\perp}$ and $A_{\perp}$ resulted in only a very small change in the computed spectra [12].

The following distributions for $g_{\|}$and $A_{\|}$will result from equations (4) and (6) :

$$
\begin{gathered}
P\left(g_{\|}\right)=\frac{1}{\sqrt{2 \pi} \sigma_{g_{1}}} \mathrm{e}^{-\frac{\left(g_{1}-g_{\mid}^{P}\right)^{2}}{2 \sigma_{g_{1}}^{2}}} \\
P\left(A_{\mid}\right)=\frac{1}{\sqrt{2 \pi} \sigma_{A_{1}}} \mathrm{e}^{-\frac{\left(A_{\mid}-A_{\mid}^{0}\right)^{2}}{2 \sigma_{A_{\mid}}^{2}}}
\end{gathered}
$$

with :

$$
\sigma_{g_{1}}=\frac{a}{\Delta^{2}} \sigma_{\Delta} ; \quad \sigma_{A_{1}}=\frac{c}{\Delta^{2}} \sigma_{\Delta}
$$

The effect of introducing the distributions of these spin Hamiltonian parameters will be that of creating a distribution in the resonant field positions, $H_{0}$, which are obtained by solving to the second order the spin Hamiltonian appearing in equation $(2)[12,13]$. For small changes in these parameters, $\delta g_{\|}$and $\delta A_{\|}$, the following fluctuation in $H_{0}$ will result :

$$
\delta H_{0}=\frac{\partial H_{0}}{\partial g_{\mid}} \delta g_{\mathrm{I}}+\frac{\partial H_{0}}{\partial A_{\mid}} \delta A_{\mid} \text {. }
$$

The next step is that of conceiving a theoretical model, encompassing the above mentioned distributions in $g_{\|}$and $A_{\|}$, and of finding whether the subsequently computer synthesized spectra are able to provide a best fit to the experimental spectra arising from all the different systems investigated.

It is well established that a correct simulation of the ESR spectra of $\mathrm{Cu}^{++}$complexes with 
axial symmetry, but in the absence of strain, can be performed by the following expression ([13] and Refs. therein) :

$$
S(\nu, H)=N \nu \int_{0}^{\frac{\pi}{2}} g_{1}^{2} f\left[\left(\nu-\nu_{0}\right)^{2}, \sigma_{\nu}^{\mathrm{R}}\right] \sin \theta \mathrm{d} \theta
$$

where $S(\nu, H)$ expresses the net absorption of microwave radiation, the normalization constant, $N$, takes account all the instrumental parameters ; $g_{1}^{2}$ represents the orientationdependent ESR transition probability ; $f\left[\left(\nu-\nu_{0}\right)^{2}, \sigma_{\nu}^{\mathrm{R}}\right]$ is the Gaussian lineshape, whose peak-to-peak halfwidth is $\sigma_{\nu}^{\mathrm{R}}$ ( $\mathrm{R}$ stands for residual linewidth that is mainly determined by the unresolved coupling of electron spin to ligand nuclei); $\nu_{0}$ are the resonant frequencies corresponding to the resonant field values at which the hyperfine lines are situated (we consider here frequency swept spectra, see Ref. [13]) ; finally the integral over $\theta$ takes into account the random orientation of the molecular symmetry axis of the copper complexes.

To take, now, into account the above mentioned fluctuations in $g_{\|}$and $A_{\|}$, equation (10) should be integrated over the distributed values of these two parameters; then we have

$$
S_{\text {strain }}(\nu, H)=\int S(\nu, H) F\left(g_{\|}, A_{\|}\right) \mathrm{d} g_{\|} \mathrm{d} A_{\|} .
$$

$F\left(g_{\|}, A_{\|}\right)$is taken as a bivariate normal density function which describes the Gaussian distribution of $g_{\|}$and $A_{\|}$in terms of the respective variances, $\left(\sigma_{g_{1}}\right)^{2}$ and $\left(\sigma_{A_{1}}\right)^{2}$, and of the correlation coefficient $\rho(-1 \leq \rho \leq+1)$.

By integrating equation (11), we see that the ESR spectrum, in the presence of strain, can then be synthesized by an expression of the type [13] :

$$
S_{\text {strain }}(\nu H)=N \nu \int_{0}^{\frac{\pi}{2}} g_{1}^{2} f\left[\left(\nu-\nu_{0}\right)^{2}, \sigma_{\nu}^{T}\right] \sin \theta \mathrm{d} \theta
$$

where $\sigma_{\nu}^{\mathrm{T}}$ can be cast into the form :

$$
\left(\sigma_{\nu}^{\mathrm{T}}\right)^{2}=\left(\sigma_{\nu}^{\mathrm{R}}\right)^{2}+\left(\sigma_{\nu}^{\mathrm{S}}\right)^{2}
$$

and

$$
\left(\sigma_{\nu}^{\mathrm{S}}\right)^{2}=\left(\frac{\partial \nu_{0}}{\partial g_{\|}}\right)^{2} \sigma_{g_{\mathrm{I}}}^{2}+\left(\frac{\partial \nu_{0}}{\partial A_{\|}}\right)^{2} \sigma_{A_{\mathbf{1}}}^{2}+2 \rho\left(\frac{\partial \nu_{0}}{\partial g_{\|}}\right)\left(\frac{\partial \nu_{0}}{\partial A_{\|}}\right) \sigma_{g_{\mid}} \sigma_{A_{\mathbf{l}}}
$$

From equation (14), $\sigma_{\nu}^{\mathrm{S}}$ can be expressed as a power expansion of $m_{I}$ and $H$ as follows :

$$
\left(\sigma_{\nu}^{\mathrm{s}}\right)^{2}=\left(\frac{g \beta}{h}\right)^{2}\left(A m_{I}^{2}+B H m_{I}+C H^{2}\right) .
$$

Equations (13) and (15) indicate that the presence of strain effects results in an $m_{I}$-dependent contribution, expressed by $\sigma_{\nu}^{\mathrm{S}}$, to the total linewidth, $\sigma_{\nu}^{\mathrm{T}}$, (which is actually observed in the experimental spectra). It should be remarked that expression (15), analitically derived in the present case, has been sometimes introduced as an empirical expression in the fit of the copper complex spectra (see Refs. reported in [12, 13, 20]. Moreover, such an additional line broadening is dependent on the magnetic field value and leads to asymmetry in the ESR line [13]. 
Finally, to compute the field swept ESR spectra that are usually recorded at a fixed frequency $\nu_{c}$ and displayed as the first derivative of the absorption, it is necessary to differentiate equation (12), taken at the frequency $\nu_{c}$, with respect to $H[13]$ :

$$
I_{\text {strain }}^{\text {th }}\left(H_{i}, p\right)=\frac{\mathrm{d} S_{\text {strain }}\left(\nu_{\mathrm{c}}, H\right)}{\mathrm{d} H} .
$$

This model function, which is non linear in the parameters $p$, has been used to fit all the experimental spectra obtained. The set of parameters $p$, with respect to which expression [1] was minimized, is : $g_{\|}, g_{\perp}, A_{\|}, A_{\perp}$, the residual linewidth $\sigma_{\nu}^{\mathrm{R}}, \sigma_{g_{\|}}, \sigma_{A_{\|}}$and $\rho$.

Pattern $\mathrm{b}$ of each figure shows the simulated ESR spectrum that best fits the corresponding experimental spectrum (pattern a). As can be seen, the agreement between each two related spectra is very good ; the goodness of the fits has been checked with the chi-square method and values ranging from 260 to 320 were obtained for $\chi^{2}$. The fitting parameters are reported in the legend of each figure. In this connection, from equation (8) it is expected that the ratio $\sigma_{A_{1}} / \sigma_{g_{1}}$ were about $P$, i.e. about $0.036 \mathrm{~cm}^{-1}$. However, while such a value is reasonably approached in the simulated spectra of figures 3 and 4 and in agreement with the values reported in reference [20], a value about five fold higher is obtained in the case of the two proteins. Such a discrepancy might be attributed either to the lower resolution achieved in the spectra of these systems or to different microenvironment sensed by the copper ions. We believe, however, that this aspect could be an interesting point to take into consideration for further refinement of the model.

Nevertheless the results obtained confirm, in our opinion, the newly emerging picture that glasses and proteins have many features in common. Their properties are characteristic for the presence of disorder on a microscopic scale. Such a disorder reflects the fact that both systems possess an immense configurational space and similar probability distributions of conformational states results for glasses and proteins.

We have, additionally, submitted all the systems studied to a careful ESR analysis as a function of the temperature (in the range from 240 down to $4 \mathrm{~K}$ ) to ascertain if a critical temperature for the onset of the spectral strain exists and/or if the latter shows some sort of dependence on the temperature itself.

We found that the strain effects in the ESR spectra arise below $200 \mathrm{~K}$ for the metalloprotein solutions and below the glass transition temperature (situated in the range from 160 to $200 \mathrm{~K}$ [15]) for the various amorphous systems investigated. Incidentally, for some of the latter systems, that also possess a polycrystalline phase, we observed that the strain effects were completely removed when the matrices switched to that phase, as a consequence of the stress relieving [15].

For protein aqueous solutions, the temperature of $200 \mathrm{~K}$ corresponds to the freezing of the conformational substates $[2,8]$ which, very probably, is consequent on the freezing of the hydration water $[1,4,7]$. For the amorphous systems the glass transition corresponds to the dynamical arrest of the structure in a metastable state in which the transport properties are drastically reduced [10].

Moreover, no significant changes were observed as the temperature was lowered progressively down to $4 \mathrm{~K}$. According to our model, the temperature independence of the spectral strain is indicative of the fact that the distributions in $g_{\|}$and $A_{\|}$do not change with the temperature below a critical value. Such a value corresponds to the onset of dynamical restrictions in our systems, in particular to the suppression of transitions among the conformational substates in proteins [8] and to the frozen-in of all the atomic motions in the glasses [10]. 
Finally, we would point out that, owing to its high sensitivity in detecting microenvironmental fluctuations around paramagnetic probes, ESR spectroscopy can be a rewarding tool to study the structural and dynamical properties of these disordered systems especially as regards the energy distribution of their substrates, the related energy barriers and their phase transitions.

\section{Acknowledgments.}

This work has been supported by CNR and MPI grants. Very useful discussions with Drs. P. Marzola and G. Giugliarelli are acknowledged.

\section{References}

[1] Singh G. P., Schink H. J., Lohneysen H. V., Parak F. and Hunklinger S., Z. Phys. B 55 (1984) 23.

[2] Ansari A., Berendzen J., Bowne S. F., Frauenfelder H., Iben I. E. T., Sauke T. B., SHYAMSUNDER E. and Young R. D., PNAS 82 (1985) 5000.

[3] STEIN D. L., PNAS 82 (1985) 3670.

[4] Doster W., Bachleitner A., Dunau R., Hiebl M. and Luscher E., Biophys. J. 50 (1986) 213.

[5] Yang I. S. and Anderson A. C., Phys. Rev. B 34 (1986) 2942 ; Phys. Rev. B 35 (1987) 9305.

[6] Schulte A. and Murray R., Phys. Rev. B 36 (1987) 1772.

[7] Elber R. and Karplus M., Science 235 (1987) 318.

[8] Frauenfelder H., Amorphous and Liquid Materials, Eds. E. Luscher et al. (Martinus Nijhoff Publ., Dordrecht) 1987.

[9] Kohler W., Friedrich J. and Scheer H., Phys. Rev. A 37 (1988) 660.

[10] Amorphous Solids : Low Temperature Properties, Ed. W. A. Phillips (Springer, Berlin) vol. 24 (1981).

[11] Stanley H. E. and Teixeira J., J. Chem. Phys. 73 (1980) 3404.

[12] Givgliarelli G. and Cannistraro S., Chem. Phys. 98 (1985) 115.

[13] Cannistraro S. and Giugliarelli G., Mol. Phys. 58 (1986) 173.

[14] Wang C. X., Giugliarelli G., Cannistraro S. and Fini C., Nuovo Cimento D 8 (1986) 76.

[15] Cannistraro S., Giugliarelli G., Marzola P. and Sacchetti F., Solid State Commun. 68 (1988) 369.

[16] Givgliarelli G. and Cannistraro S., Nuovo Cimento D 4 (1984) 194.

[17] Givgliarelli G., Tancini P. and Cannistraro S., Rev. Sci. Instrum. 58 (1987) 113.

[18] FEE J. A., Structure Bonding Berlin 23 (1975) 1.

[19] BRILl A. S., Transition Metals in Biochemistry (Springer Verlag, New York) 1977.

[20] Froncisz W. and Hyde J. S., J. Chem. Phys. 73 (1980) 3123, and references therein.

[21] Hagen W. R., J. Magn. Res. 44 (1981) 447.

[22] Hagen W. R., Hearsen D. O., Sands R. S. and Dunham W. R., J. Magn. Res. 61 (1985) 220.

[23] Hagen W. R., Hearsen D. O., Harding L. J. and Dunham W. R., J. Magn. Res. 61 (1985) 233.

[24] Hearsen D. O., Hagen W. R., Sands R. S., Grande H. J., Crespi H. L., Gunsalus I. C. and DunHaM W. R., J. Magn. Res. 69 (1986) 440.

[25] More C., Bertrand P. and Gayda J. P., J. Magn. Res. 73 (1987) 13.

[26] Fiamingo F. G., Brill A. S., Hampton D. A. and Thorkildsen R., Biophys. J. 55 (1989) 67.

[27] Abragam A. and Bleaney B., EPR of Transition Ions (Oxford Uni. Press, Oxford) 1977.

[28] BRILl A. S., Biophys. J. 22 (1978) 139.

[29] BACCI M., private communication.

[30] Kivelson D. and Neiman R., J. Chem. Phys. 35 (1961) 149. 